\title{
DOENÇA DE CHAGAS
}

Renato Maurício de Oliveira, ${ }^{I}$ Ana Lúcia Sampaio Sgambatti de Andrade ${ }^{2}$ e Celina Maria Turchi Martelli ${ }^{2}$

\section{RESUMO}

Ensaios clínicos duplo-cegos, aleatorizados, são considerados o paradigma da medicina baseada em evidência. Em 1991, iniciou-se um ensaio de campo aleatório, duplo-cego, placebo controlado, em áreas rurais do nordeste do Estado de Goiás, para avaliar a eficácia do benzonidazol no tratamento de crianças de 7 a 12 anos soropositivas ao Trypanosoma cruzi. A experiência de conduzir um ensaio de campo em áreas rurais e endêmicas para doença de Chagas, envolvendo não só os aspectos relacionados à aderência ao medicamento mas também a necessidade de manutenção de controle vetorial em grandes áreas, tornou a investigação operacionalmente complexa e merecedora de ser relatada. Este artigo descreve os aspectos mais relevantes referentes à condução do trabalho de campo em ensaio terapêutico para doença de Chagas e discute as principais questð̃es metodológicas deste tipo de investigação.

UNITERMOS: Ensaio clínico. Doença de Chagas. Epidemiologia. Metodologia.

\section{INTRODUÇÃO}

Os ensaios clínicos controlados são considerados os delineamentos ideais para fornecer evidências do efeito de intervenções em saúde, pois são os estudos que mais se aproximam de um experimento laboratorial, passível de ser conduzido em seres humanos. Ensaios aleatórios, placebos controlados e duplo-cegos ("Double Blind-Randomized Controlled Trial"-DB-RCT), constituem o paradigma da medicina baseada em evidências. As diferenças

1 Professor Assistente do Departamento de Medicina Tropical, Saúde Coletiva e Dermatologia (DMTSCD) do Instituto de Patologia Tropical e Saúde Pública (IPTSP) da Universidade Federal de Goiás (UFG).

2. Professoras Titulares do DMTSCD, IPTSP, UFG.

Endereço para correspondência: Rua Delenda Rezende de Melo esq. com $1^{\text {a }}$ Avenida, Setor Universitário. Caixa Postal 131, CEP 74605-050, Goiânia, GO. 
fundamentais entre os estudos experimentais e os observacionais consistem na alocação da intervenção pelo investigador e no processo de aleatorização dos participantes do ensaio clínico para receber a intervenção testada versus grupo de comparação. $O$ processo de aleatorização visa tornar os grupos estudados comparáveis em relação a todas as variáveis de confusão conhecidas ou não, considerando-se que o tamanho do estudo seja adequado. Esta similaridade inicial entre os grupos possibilita que as diferenças encontradas ao final do monitoramento sejam decorrentes da intervenção, desde que, na condução da investigação, não sejam introduzidos vieses (Pocock, 1983; Hulley \& Cummings, 1988; Smith \& Morrow, 1998).

Ensaios terapêuticos em doenças transmissíveis apresentam grande impacto em saúde pública, pois, comprovada a eficácia da droga estudada, o tratamento da doença pode interferir na cadeia de transmissão, reduzindo a incidência do evento na comunidade, diferentemente das doenças não transmissíveis (Smith \& Morrow, 1998). Embora haja consenso sobre a importância dos DB-RCT na avaliação de novas drogas antecedendo a sua utilização na prática clínica e em saúde pública, este tipo de avaliação em doenças endêmicas, como por exemplo na doença de Chagas, tem sido pouco contemplado na literatura científica da última década (Dias \& Coura, 1997). Alguns fatores, que incluem a formulação da hipótese de estudo pela identificação da lacuna existente na literatura, os aspectos operacionais de implementação do trabalho de campo, a manutenção de equipes de investigadores multidisciplinares e experientes, o financiamento e questões de natureza ética, podem ser listados como potenciais dificuldades na realização de ensaios clínicos do tipo DB-RCT.

Foi iniciado em 1991, em áreas rurais do nordeste do Estado de Goiás, um ensaio de campo aleatório, duplo-cego, placebo controlado, para avaliar a eficácia do benzonidazol no tratamento de crianças de 7 a 12 anos soropositivas ao Trypanosoma cruzi (Andrade et al., 1996; Spadeto, 1999). A experiência de conduzir um ensaio de campo em áreas rurais e endêmicas para doença de Chagas, envolvendo não só a administração de medicamentos mas também a necessidade de controle vetorial em grandes áreas, tornou a investigação operacionalmente complexa e merecedora de ser relatada. Este artigo propõe-se a descrever os aspectos mais relevantes referentes à condução do trabalho de campo em ensaio terapêutico para doença de Chagas e discutir as principais questões metodológicas deste tipo de investigação.

\section{CONSIDERAÇÕES METODOLÓGICAS}

Escolha da área de estudo

Uma questão fundamental ao se delinear um ensaio de campo é estabelecer critérios para escolha do local para recrutamento dos participantes. Nesta investigação, a área para desenvolver o projeto deveria 
apresentar alta prevalência de infecção pelo $T$. cruzi e ausência de transmissão vetorial pelo $T$. infestans para assegurar a não-reinfecção dos participantes durante o período de estudo. Considerando-se também que este ensaio clínico previa avaliações sucessivas e acompanhamento prolongado dos participantes, era necessário que a população fosse relativamente estável do ponto de vista migratório (Andrade \& Zicker, 1997).

Para seleção da área de estudo foram utilizadas informações do inquérito sorológico de prevalência de infecção chagásica no Brasil (Camargo et al., 1984) e de taxas de infestação por triatomíneo de $42 \%$ na área de acordo com inquérito nacional realizado no período de 1975 a 1983 (Silveira et al., 1984). Não se identificou de imediato, com as informações disponíveis, uma região que apresentasse características ideais para realização do estudo. Embora a soroprevalência global de Goiás, identificada no inquérito nacional, fosse de 7,4\%, os municípios de Posse, Simolândia e Guarani de Goiás apresentavam taxas entre $12 \%$ e $23 \%$ na faixa etária de 7 a 14 anos na década de 1970. Ressalta-se que os dados publicados são geralmente apresentados de forma agregada, o que implica em valores médios de prevalência, mascarando áreas de maior e menor morbidade. Além disso, estudos conduzidos no início da década de 1990 (Andrade et al., 1995 b) detectaram alta infestação domiciliar (12,6\%) pelo Triatoma infestans na região, o que provavelmente configuraria áreas de alta morbidade pelo $T$. cruzi.

Foi necessário incluir no protocolo de pesquisa medidas de controle vetorial, com uso de inseticidas durante todo o estudo, para impedir a transmissão da infecção no decorrer do ensaio de campo. Nesta etapa foi fundamental a participação de profissionais da Fundação Nacional de Saúde (FNS) e a incorporação de um entomologista na equipe de campo para propiciar intensa vigilância do triatomíneo na região investigada. A comunidade foi treinada para identificação de triatomíneos e conscientizada da importância de manter um controle da reinfestação das moradias, passando a ser uma forte aliada no combate ao vetor. Foram também orientados para notificar imediatamente à FNS a presença de triatomíneos nas habitações.

Organização e gerenciamento do trabalho de campo

O planejamento e a condução adequados das atividades de campo são essenciais para a obtenção de dados confiáveis e para o sucesso do ensaio. O coordenador de campo ou gerente é o responsável pelo desenvolvimento da investigação nos seus aspectos operacionais. De acordo com Farrell (1998), o coordenador de campo é o elemento-chave, responsável pela gerência do ensaio no dia-a-dia, o que requer a profissionalização deste tipo de atividade. $\mathrm{O}$ investigador principal e o coordenador de campo discutem as estratégias operacionais e os procedimentos de campo. As características de um coordenador de campo incluem o conhecimento técnico 
e qualidades pessoais de liderança, para ser capaz de gerenciar a equipe de campo e garantir a padronização e qualidade dos dados coletados. Em nosso meio, geralmente os membros da equipe incorporam múltiplas atividades inerentes à etapa de campo da investigação. Neste estudo, a equipe de campo foi constituída de dois profissionais para execução da maioria das atividades, incluindo a coleta de material biológico, e respectiva conservação, e ainda a realização de eletrocardiogramas e o levantamento entomológico sob supervisão. Ressalte-se que durante todo o desenvolvimento do projeto a participação de técnicos da Fundação Nacional de Saúde (Regional de Formosa) foi decisiva, visto que estes profissionais detêm informações detalhadas da região em estudo, até mesmo com croquis das casas e das escolas.

A viagem inicial para a área de estudo foi de reconhecimento e de realização de contatos com os profissionais de saúde, com identificação dos hospitais, postos e laboratórios, que serviram de referência regional para realização de exames laboratoriais. A Secretaria Municipal de Educação forneceu as informações referentes ao número de escolas e alunos da zona rural. Foram feitos contatos com os agentes de saúde das comunidades rurais, líderes locais e com as professoras das escolas discutindo-se os objetivos do trabalho e solicitando colaboração para realizar as atividades.

Inicialmente, encontrou-se resistência por parte dos médicos locais em colaborar com as atividades do projeto, com alguns relatos de que pesquisadores anteriores não divulgaram os resultados regionalmente. Esta dificuldade foi superada com o compromisso da equipe de conduzir o trabalho em conjunto com os profissionais de saúde da região.

A relação de confiança e empatia que se estabeleceu com a comunidade foi fundamental para o desenvolvimento do projeto. Procurou-se informar a população de maneira adequada e simplificada sobre os objetivos da investigação e todos os resultados dos exames realizados (sorologias, eletrocardiogramas) foram entregues aos participantes com explicações sobre a interpretação e encaminhamentos necessários aos serviços de saúde. Levouse em conta que o cumprimento da agenda de compromissos é parte obrigatória de qualquer investigação de campo, assim como o atendimento aos preceitos éticos assumidos com a comunidade.

O cronograma de trabalho é essencial em zonas rurais em países como o Brasil. Por isso, devido às péssimas condições das estradas, as atividades foram planejadas, evitando-se viajar para a área de estudo em períodos chuvosos ou de férias escolares.

Recrutamento de participantes e amostragem

Os procedimentos operacionais para selecionar os participantes de ensaios clínicos conduzidos em áreas rurais são geralmente complexos, e dependem, boa parte das vezes, da criatividade dos investigadores para se 
otimizar o custo-beneficio da investigação, especialmente levando-se em consideração a baixa densidade populacional nestas regiões. Considerando-se que a população-alvo era predominantemente de comunidades rurais, a estratégia adotada foi utilizar as escolas como local de recrutamento dos participantés do ensaio clínico (Figura 1). Triagens sorológicas de crianças ou escolares têm sido a metodologia utilizada para avaliação da efetividade dos Programas de Controle da doença de Chagas em várias localidades rurais nas últimas décadas (Souza et al., 1984; Hoff et al., 1985; Carneiro \& Antunes, 1994; Costa et al., 1998). Dentre as vantagens deste tipo de abordagem, destacam-se: a facilidade no recrutamento das crianças, o monitoramento adequado na administração da droga e o acompanhamento com apoio dos professores locais. No entanto, a adoção desta estratégia implicou na inclusão de múltiplos locais de recrutamento, num total de 60 escolas de 46 localidades rurais, o que tornou o trabalho de campo mais complexo.

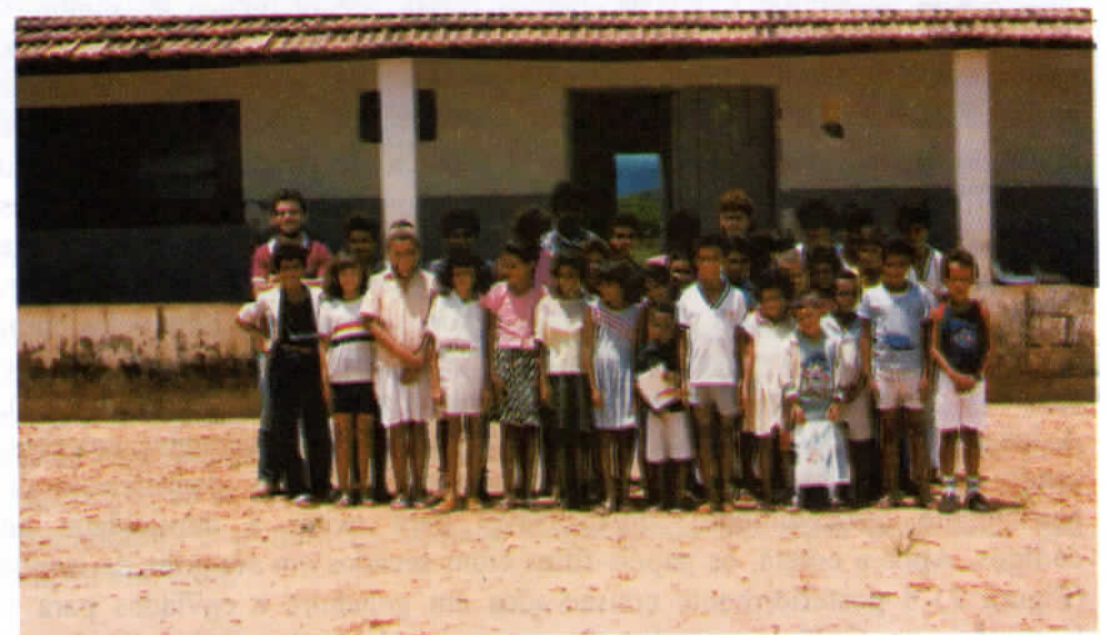

Figura 1. Alunos de escola rural do município de Posse

Habitualmente, nos ensaios de campo, é selecionado um número maior de participantes que o previsto no tamanho da amostra, devido às perdas que ocorrem durante o período de seguimento dos grupos (Pocock, 1983). A amostragem para triagem sorológica dos participantes do ensaio clínico foi calculada levando-se em conta uma prevalência de infecção pelo T. cruzi de $2 \%$ em escolares, de acordo com estudos realizados na última década. Assim, para selecionar os 130 participantes potencialmente elegíveis para o ensaio terapêutico, era necessário rastrear aproximadamente 8.000 crianças. No entanto, durante o estudo piloto em uma das comunidades da área selecionada para o estudo, detectou-se $8 \%$ de soropositividade na faixa etária de 7 a 12 anos. Desta forma, a investigação laboratorial de 1.990 
crianças foi suficiente para identificar os indivíduos elegíveis para o ensaio clínico (Andrade et al., 1992) e permitir a generalização dos resultados obtidos (Hennekens \& Buring, 1987). A amostragem correspondeu a $82 \%$ da população de crianças de 7 a 12 anos residentes na área de estudo.

Esta informação sobre a população da qual os participantes são recrutados, com as respectivos critérios de inclusão e exclusão, são geralmente publicados como perfil do ensaio, possibilitando identificar a validade interna da amostra estudada e construir um juizo de valor sobre a validade externa do estudo, isto é, extrapolar os resultados para outras populações (Sackett, 1979; Martelli \& Andrade, 1997).

Procedimentos na coleta de dados e no estudo clínico-laboratorial

Procedimentos de triagem servem para identificar doença/infecção, através de testes, exames ou outros métodos. Para ser usado em triagens sorológicas de campo um teste deve apresentar pelo menos as seguintes características: ser simples e rápido de realizar, causar o mínimo possivel de desconforto, apresentar facilidade de armazenamento e de transporte e alta sensibilidade (Galen \& Gambino, 1975; Pereira \& Andrade, 1997). Para tanto, nesta investigação, utilizou-se a técnica de coleta em papel-filtro (Guimarães, 1984) para detectar os soropositivos para T. cruzi. Por questões operacionais, no intuito de facilitar e agilizar a coleta de sangue, procedeu-se da seguinte maneira: utilizou-se papel-filtro Whatman® número 1 , circular, $90 \mathrm{~mm}$, dividido em duas partes iguais, sendo que em cada uma delas eram delimitados a lápis, com uso de um compasso, dois círculos de $16 \mathrm{~mm}$ de diâmetro (área de $2 \mathrm{~cm}^{2}$ ), metade da área preconizada por Guimarães (1984). A identificação dos papéis-filtro foi feita utilizando-se etiquetas colantes, sendo uma duplicata da mesma fixada numa ficha de identificação do participante, procedimento indispensável para evitar a duplicidade de códigos. Após a coleta, os papéis-filtro eram secados em suportes de isopor (Figura 2) e posteriormente conservados em geladeira e enviados para o laboratório em Goiânia em caixa térmica com gelo, onde eram realizados os exames sorológicos. A equipe para a coleta de sangue era composta basicamente de quatro técnicos, dois deles na identificação das crianças e dois para coleta. Em cada semana de trabalho era possivel coletar uma média de 300 amostras de sangue através de punção digital. Diariamente programava-se um roteiro a ser seguido, $\mathrm{e}$ as crianças eram puncionadas nas escolas, onde as fichas eram simultaneamente preenchidas. 


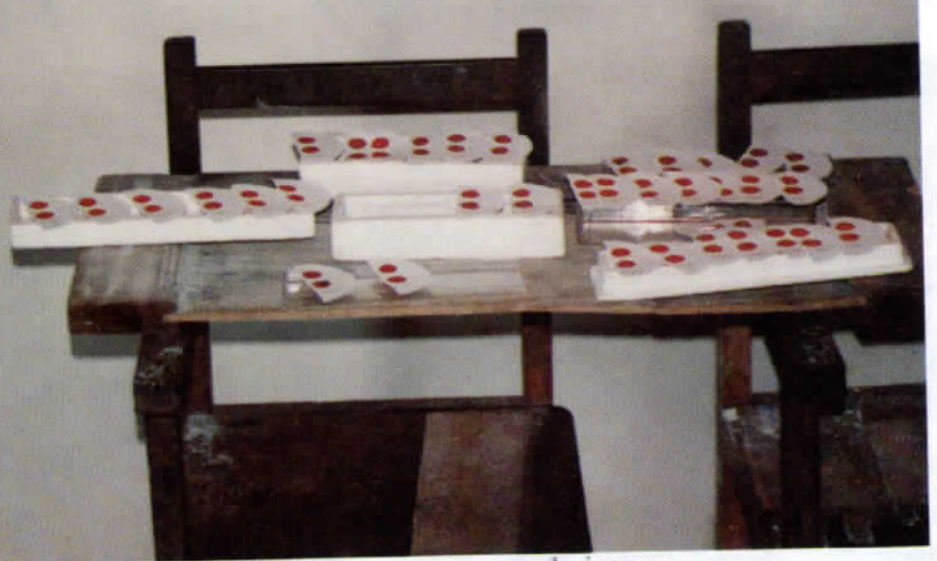

Figura 2. Secagem dos papéis-filtro em suportes de isopor

Realizou-se a coleta de sangue venoso $(10 \mathrm{ml})$ para confirmação da soropositividade em 150 crianças, utilizando-se o sistema Vacutainer® (Figura 3), ideal pela facilidade de manuseio, conservação e transporte. Ao final do dia esse sangue era centrifugado para separação do soro, pelo coordenador de campo. As crianças eram previamente avisadas da coleta de sangue e aquelas que não compareciam à escola eram visitadas em suas casas, para realizar a coleta. As amostras de sangue eram identificadas com etiquetas e, ao se fazer a separação do soro, os tubos eram identificados com esparadrapo, e o número anotado empregando-se caneta com tinta não solúvel em água.

Administração do medicamento

Nos ensaios de campo com característica duplo-cego e placebo controlado, o preparo/manipulação do placebo corresponde a uma etapa de fundamental importância para garantir o mascaramento durante a administração da intervenção e avaliação dos efeitos adversos. Os comprimidos administrados como placebo foram manipulados pela Central de Medicamentos de Goiás (CEME-GO), incluindo na sua composição todos os ingredientes contidos no Benzonidazol (BZ), à exceção do princípio ativo. A forma, a cor, a ranhura e o sabor do placebo se assemelharam aos do BZ, garantindo-se a não-identificação da droga, tanto para quem administra quanto para quem recebe, para evitar o viés de observador/participante nas avaliaçס̃es clínicas e laboratoriais (Sackett, 1979; Hulley \& Cummings, 
1988). Uma lista de alocação aleatória com código numérico foi previamente preparada por um observador externo ao projeto. Para assegurar o controle de qualidade, os comprimidos de $\mathrm{BZ}$ e placebo foram enviados de maneira codificada para o laboratório de referência do Ministério da Saúde para confirmar a composição química dos mesmos.

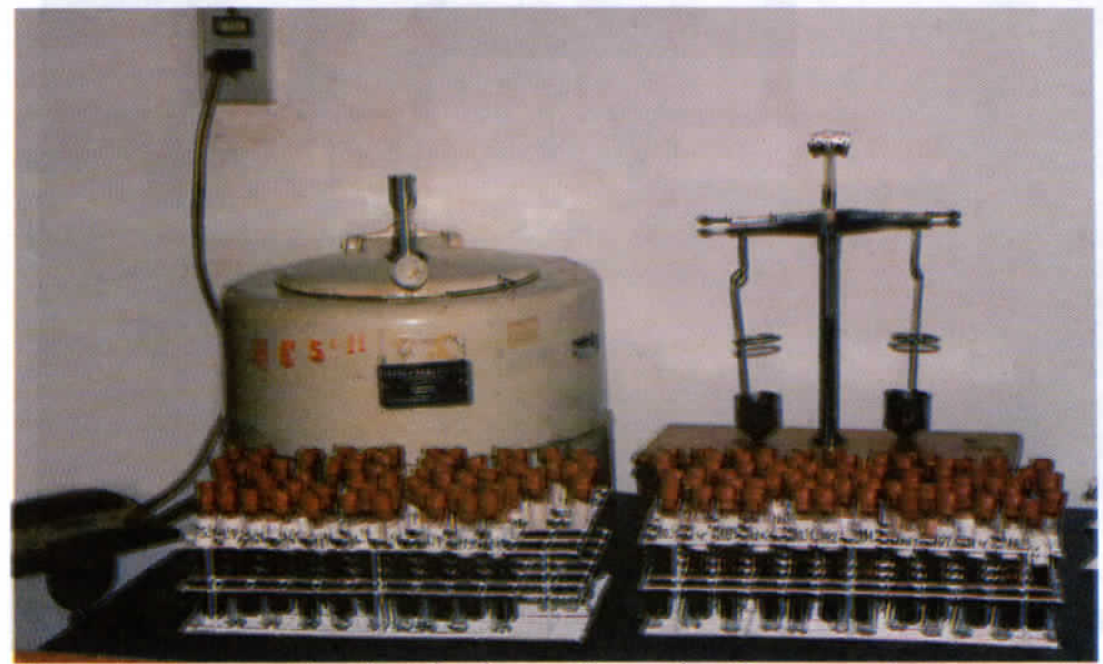

Figura 3. Sangue coletado pelo sistema Vacutainer(8

Devido à duração de 60 dias do regime terapêutico e o fato de as crianças estarem em idade escolar, a estratégia adotada foi treinar os professores para administrar o tratamento. Para cada criança um observador externo preparava um recipiente plástico contendo o número total dos comprimidos, com posologia de acordo com o peso. As crianças recebiam a medicação na sala de aula, e nos finais de semana e feriados levavam o medicamento para casa. Os efeitos colaterais eram monitorados diariamente em uma ficha individual. Qualquer efeito adverso grave era comunicado imediatamente por telefone ao centro coordenador e definida a orientação necessária para a continuidade, a suspensão do tratamento e/ou a quebra do código. Em apenas um caso foi necessária a suspensão da droga, reforçando as evidências clínicas de que o tratamento da infecção pela $T$. cruzi com BZ em crianças é bem tolerado (Rassi \& Luquetti, 1992; Andrade et al., 1996; Estani et al., 1998). Possíveis efeitos cardíacos adversos do BZ foram monitorados por eletrocardiógrafo, utilizando-se uma maca portátil (Figura 4). 


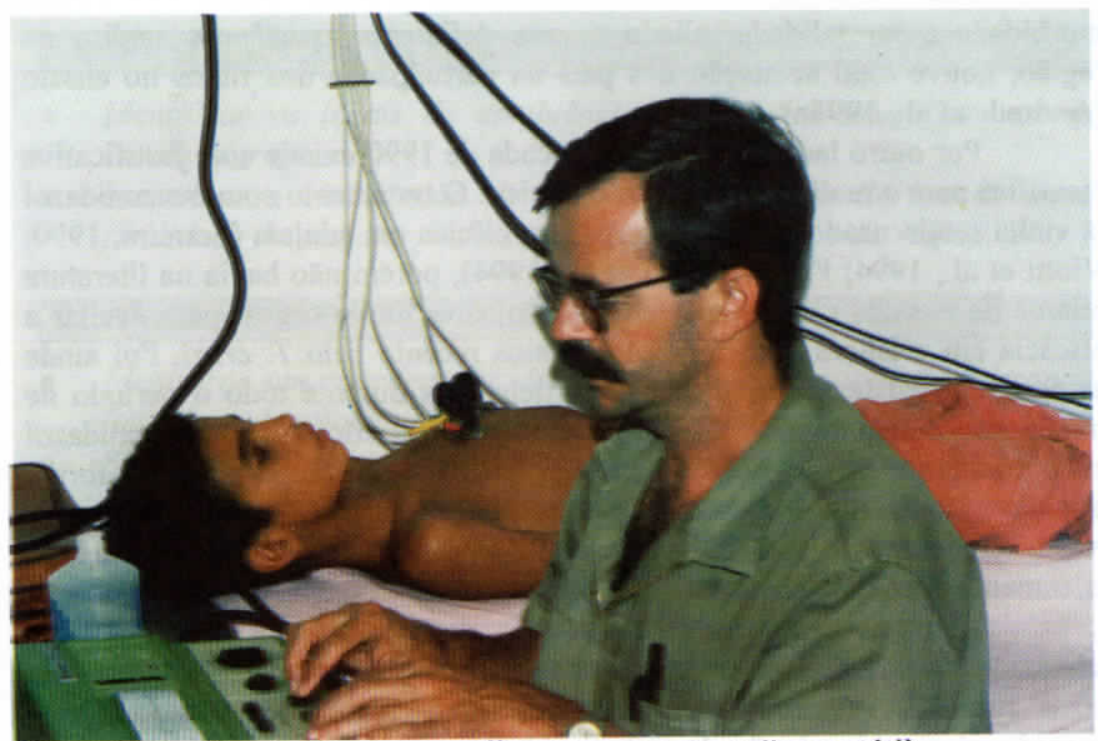

Figura 4. Realização de eletrocardiograma com aparelho portátil

Procedimentos éticos

O conhecimento sobre os mecanismos de ocorrência das doenças baseado apenas em trabalhos com modelos em animais de laboratório ou estudos fisiológicos é insuficiente para conclusões a respeito da eficácia destes procedimentos no ser humano; daí a necessidade de ensaios clínicos controlados (Fletcher et al., 1989). Neste tipo de estudo as questões éticas são de fundamental importância, e os projetos devem necessariamente ser submetidos e aprovados por Comitês de Ética como pré-requisito para o financiamento, garantindo-se a voluntariedade, a confidencialidade e uma investigação com danos minimizados aos participantes (Council for International Organizations of Medical Sciences, 1993; Ministério da Saúde, Brasil, 1997).

O consentimento informado é um documento assinado pelos participantes ou responsáveis, que fica arquivado nos protocolos individuais (WHO, 1995). Este tipo de documentação deve ser arquivado em papel por um período mínimo de 10 anos, como parte do requerimento ético. Neste estudo, todos os pais foram informados sobre os objetivos do projeto, sobre os potenciais benefícios para o indivíduo e para a comunidade e prováveis efeitos colaterais do medicamento, bem como sobre a liberdade de poder retirar o filho do ensaio a qualquer momento. As crianças soropositivas elegíveis foram convidadas a participar da investigação e o consentimento informado foi assinado pelos responsáveis. Considerando-se que a população local tinha conhecimento da doença de Chagas como importante causa de 
morbidade e mortalidade, aliado a uma deficiente assistência médica na região, houve total aceitação dos pais na participação dos filhos no ensaio (Andrade et al., 1995a).

Por outro lado, no início da década de 1990 existia uma justificativa científica para a realização do ensaio clínico. $O$ tratamento com benzonidazol já vinha sendo usado no Brasil na prática clínica em adultos (Ferreira, 1990; Viotti et al., 1994; Fragata Filho et al., 1994), porém não havia na literatura relatos de ensaios clínicos placebo-controlados, duplo-cegos, para avaliar a eficácia em crianças com infecção crônica recente pelo $T$. cruzi. Foi ainda assegurada assistência médica aos participantes durante todo o período de tratamento e seguimento. Diante da evidência de eficácia do benzonidazol encontrada na investigação, todas as crianças do grupo-placebo, foram tratadas com BZ após o término do ensaio conforme previsto no protocolo. Neste contexto, o uso de placebo no ensaio clínico foi considerado eticamente adequado.

Algumas recomendações são fundamentais para viabilizar um adequado desenvolvimento de um ensaio clínico de campo conduzido em áreas rurais. A seguir, procurou-se relacionar alguns aspectos a serem considerados na condução do trabalho de campo, sob forma de um roteiro básico.

\section{ROTEIRO PARA DESENVOLVIMENTO DE ATIVIDADES DE CAMPO DE UM ENSAIO CLINICO}

\section{VISITA PREVIA A PROVAVEL REGIAO DO ESTUDO}

- Proceder ao reconhecimento da área para verificar a factibilidade do estudo em termos técnicos e logísticos.

- Realizar contato com as Secretarias de Saúde elou Educação da região.

- Identificar os líderes da região, particularmente dentre os agentes de saúde.

- Verificar a disponibilidade dos serviços de saúde locais, postos de saúde, hospitais, laboratórios para dar suporte à investigação.

- Checar as informações/registros sobre a enfermidade: incidência, prevalência, sazonalidade, casos assintomáticos/subclínicos.

- Avaliar a aceitabilidade da intervenção pela população-alvo e a comunidade em geral.

\section{ESCOLHA DA AREA DE ESTUDO}

- Selecionar população relativamente estável, com baixa taxa de migração e preferentemente com alta prevalência do evento.

- Viabilizar a parceria da população na execução do estudo.

- Atuar de forma conjunta com os serviços de saúde locais/regionais. 
- Identificar os locais de confluência dos participantes (por exemplo escolas, igrejas).

- Incentivar a cooperação de grupos comunitários de acordo com a finalidade do projeto, professores e agentes de saúde da região.

\section{ORGANIZAÇÃO DO TRABALHO DE CAMPO}

- Identificar um coordenador de campo com capacidade de liderança e facilidade de relacionamento com a comunidade.

- Selecionar e treinar a equipe para execução das atividades.

- Planejar adequadamente as atividades, levando em consideração as férias escolares, feriados, periodos chuvosos etc.

- Realizar estudo piloto para avaliar a adequação dos instrumentos de coleta e proceder aos ajustes necessários.

- Contactar os profissionais de saúde da região e discutir aspectos técnico-operacionais da investigação.

- Utilizar, sempre que possivel, a infra-estrutura dos Programas de Controle locais.

- Garantir o cumprimento da agenda de compromissos com a comunidade de maneira oportuna e respeitosa.

\section{TRIAGEM E SEGUIMENTO DOS PARTICIPANTES}

- Utilizar técnicas de coleta adequadas para o campo e material de fácil conservação.

- Proceder à organização de uma lista de material necessário para coleta e identificação das amostras: luvas, etiquetas, canetas, lápis etc.

- Viabilizar mecanismos operacionais para detectar participantes que migram da região.

\section{ADMINISTRAÇÁO DA DROGA}

- Garantir a supervisão na administração da droga.

- Identificar mecanismos eficientes para monitorar os potenciais efeitos colaterais.

- Assegurar acompanhamento médico durante todo o estudo.

\section{CONSENTIMENTO INFORMADO}

- Certificar-se da aprovação do protocolo de pesquisa por comissões de ética. 
- Elaborar uma carta objetiva e com linguagem compativel à cultura local, informando sobre o estudo.

- Esclarecer a comunidade sobre os riscos e beneficios da intervenção.

- Obter o consentimento por escrito dos participantes ou responsáveis.

- Garantir o tratamento a posteriori dos indivíduos que usaram placebo, caso a eficácia da droga seja confirmada.

\section{CONSIDERAÇÕES FINAIS}

Este ensaio clínico conduzido durante sete anos promoveu na região uma grande mobilização de forças no sentido da vigilância e controle da doença de Chagas e culminou com medidas intensas de controle vetorial, incluindo não somente o uso de inseticidas, mas também a melhoria das habitações da região com construções de casas de alvenaria para a maioria da população (Figuras 5,6 e 7). Estas medidas levaram à interrupção da transmissão na área de estudo, fato que, além de constituir-se em inestimável ganho para a população da região, mostrou ser viável a interrupção da transmissão vetorial da doença. Os resultados desse ensaio clínico viabilizaram também a adoção do benzonidazol pelo Ministério da Saúde na rotina de tratamento em crianças/adolescentes soropositivas ao $T$. cruzi (Ministério da Saúde, 1997; Luquetti, 1997).

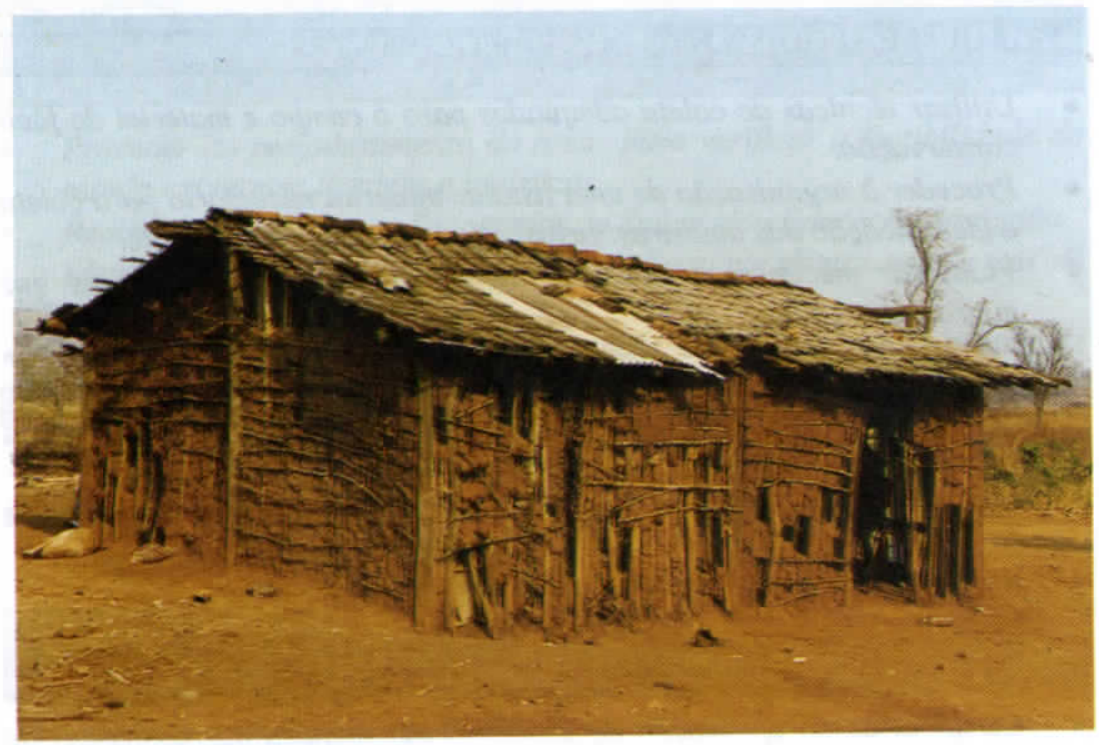

Figura 5. Moradia típica da região na área rural 


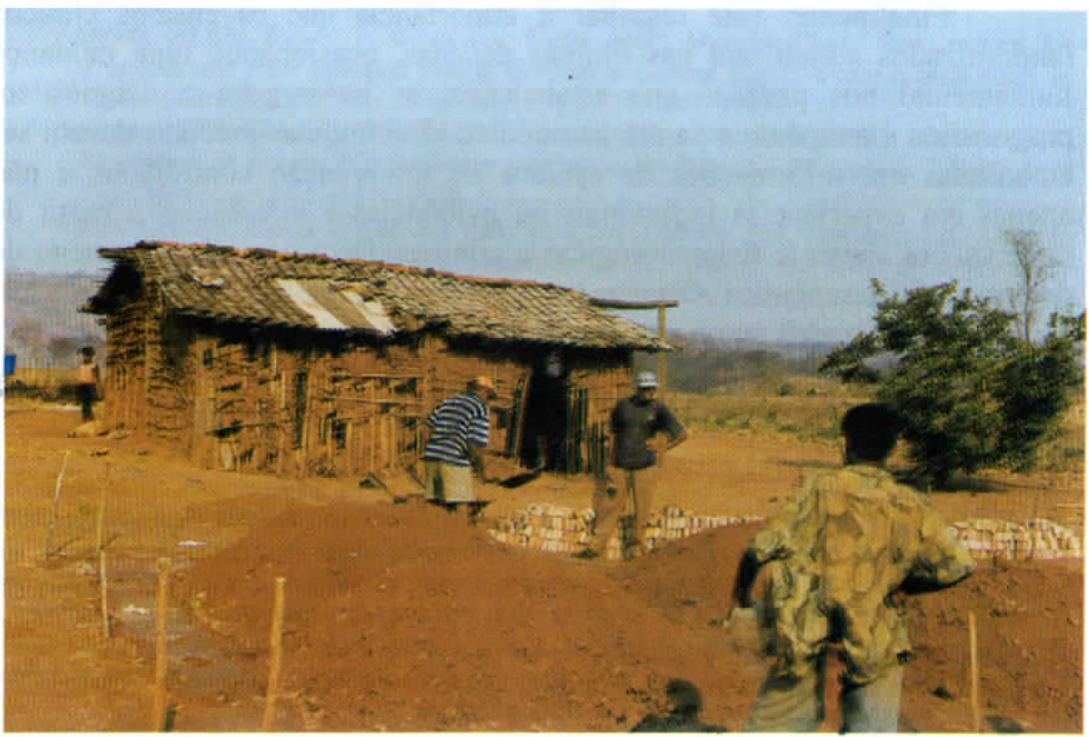

Figura 6. Casa de pau-a-pique e início de nova construção

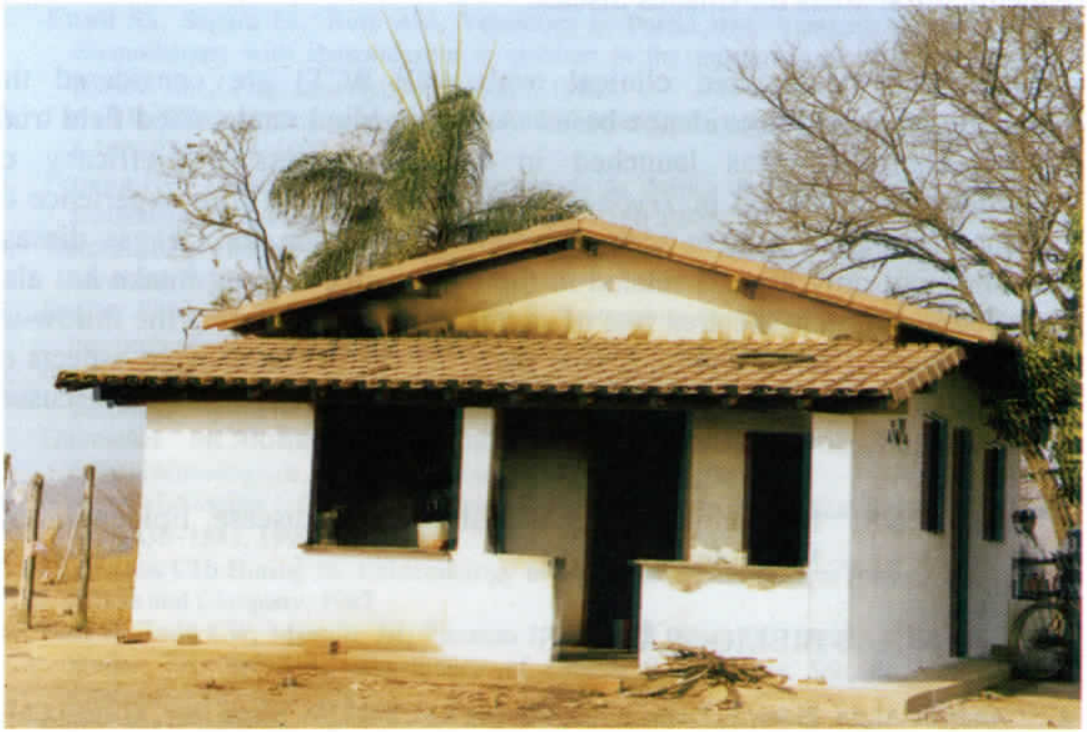

Figura 7. Casa de alvenaria, construída em substituição às casas de pau-a-pique 
Finalmente, vale ressaltar a importância que os ensaios clínicos randomizados adquiriram nas últimas décadas, ocasionando uma mudança fundamental nos padrões que estabelecem as bases para o diagnóstico, prognóstico e terapêutica na prática médica. As condutas médicas devem ser embasadas em informações de estudos de intervenção controlados e não apenas em experiências individuais ou evidências e inferências a partir de observações somente fisiopatológicas e clínicas. Decisões sobre emprego de determinada terapêutica devem ter como respaldo estudos controlados, aleatórios, realizados numa amostra representativa, com poder estatístico de detectar diferenças clinicamente significantes (Rosser, 1999; Mant, 1999; Weel \& Knottnerus, 1999; Guyatt et al., 1999; Sniderman, 1999).

\section{AGRADECIMENTOS}

Ao UNDP/Word Bank/WHO/TDR, CNPq e FUNAPE pelo financiamento da investigação. A Fundação Nacional de Saúde pela participação em todas as etapas do projeto, em especial aos técnicos da regional de Formosa, Goiás. Ao professor Ionizete Garcia da Silva pela supervisào entomológica. A Lourdes Ferreira da Mota pela colaboração na realização dos exames e eletrocardiogramas. Aos colegas Gil Eduardo Perini, Andréa Inês Spadeto Aires e João Bosco Siqueira Júnior pela contribuição nas atividades de campo do projeto. Ao professor Alejandro Luquetti Ostermayer pela realização dos testes sorológicos.

\section{SUMMARY}

Methodological and operational issues on the conduction of field trials in endemic rural areas for Chagas disease.

Double-blind randomized clinical trials (DB-RCT) are considered the paradigm of medicine evidence-based. A double-blind randomized field trial, placebo-controlled was launched in 1991 to assess the efficacy of benznidazole childhood in Trypanosoma cruzi infection. The experience of conducting a field trial in scattered population region for Chagas disease including not only aspects related to the compliance of drug intake but also the need to maintain the area free of vector transmission during the follow-up period deserved to be reported. This paper describes the relevant aspects of the field work in clinical trial using as example Chagas disease and discusses the main methodological issues of this type of investigation.

KEY WORDS: Randomized clinical trials. Chagas disease. Epidemiology. Methodology.

\section{REFERÊNCIAS BIBLIOGRÁFICAS}

1. Andrade ALSS, Zicker F, Luquetti AO, Oliveira RM, Silva SA, Souza JMP, Martelli CMT. Surveillance of Trypanosoma cruzi transmission by serological screening of schoolchildren. Bull World Health Organ 70:625-629, 1992. 
2. Andrade ALSS, Zicker F, Silva IG, Souza JMP, Martelli CMT. Risk factors for Trypanosoma cruzi infection among children in Central Brazil: a case-control study in vector control settings. Am J Trop Med Hyg 52:183-187, 1995a.

3. Andrade ALSS, Zicker F, Oliveira RM, Silva IG, Silva SA, Andrade SG, Martelli CMT. Evaluation of risk factors for house infestation by Triatoma infestans in Brasil. Am J Trop Med Hyg 53:443-447, 1995b.

4. Andrade ALSS, Zicker F, Oliveira RM, Silva SA, Luquetti AO, Travassos LR, Almeida IC, Andrade SS, Andrade JG, Martelli CMT. Randomised trial of efficacy of benznidazole in treatment of early Trypanosoma cruzi infection. Lancet 348:1407-1413, 1996.

5. Andrade ALSS, Zicker F. Ensaio Clínico. In: Andrade ALSS, Zicker F. Métodos de Investigação Epidemiológica em Doenças Transmissiveis. Organização Pan-Americana de Saúde: Fundação Nacional de Saúde, Brasília 1:93-115, 1997.

6. Camargo ME, Silva GR, Castilho EA, Silveira AC. Inquérito sorológico da prevalência da infecção chagásica no Brasil, 1975/1980. Rev Inst Med Trop São Paulo 26: 192-204, 1984.

7. Carneiro M, Antunes CMF. A quasi-experimental epidemiological model for evaluating public heath programmes: efficacy of a Chagas disease control programme in Brazil. Bull World Health Organ 72:721-728, 1994.

8. Costa FC, Vitor RWA, Antunes CMF, Carneiro M. Chagas Disease Control Programme in Brazil: a study of the effectiveness of 13 years of intervention. Bull World Health Organ 76:385-91, 1998.

9. Council for International Organizations of Medical Sciences. International ethical guidelines for biomedical research involving human subjects. Geneva, CIOMS, annex 1, 1993.

10. Dias JCP, Coura JR. Epidemiologia. In: Clinica e terapèutica da doença de Chagas: uma abordagem prática para o clinico geral. Fiocruz. Rio de Janeiro 20:33-66, 1997.

11. Estani SS, Segura EL, Ruiz AM, Velazquez E, Porcel BM, Yampotis C. Efficacy of chemotherapy with Benznidazole in children in the indeterminate phase of Chagas' disease. Am J Trop Med Hyg 59:526-529, 1998.

12. Farrell B. Efficient management of randomised controlled trials: nature or nurture. $\mathrm{Br} \mathrm{Med}$ J 317:1236-1239, 1998.

13. Ferreira HO. Tratamento da forma inderminada da doença de Chagas com Nifurtimox e Benzonidazol. Rev Soc Bras Med Trop 23:209-211, 1990.

14. Fletcher RH, Fletcher SW, Wagner EH. Epidemiologia Clinica. Porto Alegre. Artes Médicas, 1989.

15. Fragata Filho AA, Silva MAD, Boainain E. Tratamento etiológico da doença de Chagas nas fases aguda e crônica. Rev Soc Cardiol Estado de São Paulo 4:192-197, 1994.

16. Galen RS, Gambino SR. Beyond normality: the predictive value and efficiency of medical diagnoses. New York, Ed. John Wiley \& Sons, 1975.

17. Guimarães MCS. Coleta de sangue em papel-filtro para fins de inquéritos soroepidemiológicos. Rev Soc Bras Med Trop 17: 7-45, 1984.

18. Guyatt GH, Sinclair J, Cook DJ, Glasziou P. Users'guides to the medical literature. JAMA, 281:1836-1843, 1999.

19. Hennekens $\mathrm{CH}$, Buring JE. Epidemiology in Medicine. $5^{\text {th }}$ ed. Boston/Toronto, Ed. Little, Brown and Company, 1987.

20. Hoff R, Todd CW, Maguire JH, Piesman J, Mott KE, Mota EE, Sleigh A, Sherlock IA, Weller TH. Serologic surveillance of Chagas'disease. Ann Soc Belg Med Trop 65(suppl 1):187-196, 1985.

21. Hulley SB, Cummings SR. Designing clinical research. Williams \& Wilkins. Baltimore, 1988.

22. Luquetti AO. Etiological treatment for Chagas disease. Parasitology Today 13:127-128, 1997. 
23. Mant D. Can randomised trials inform clinical decisions about individual patients? Lancet 353:743-746, 1999.

24. Martelli CMT \& Andrade ALSS. Estudos de Prevalência. In: Andrade ALSS \& Zicker F. Métodos de Investigação Epidemiológica em Doenças Transmissiveis. Organização Pan-Americana de Saúde: Fundação Nacional de Saúde 1: 31-50, 1997.

25. Ministério da Saúde, Brasil. Tratamento etiológico da doença de Chagas. Fundação Nacional de Saúde. Coordenação de Controle de Doenças Transmissíveis por Vetores. Gerência Técnica de Doença de Chagas. 32p. Brasília, 1997.

26. Ministério da Saúde, Brasil. Diretrizes e normas regulamentadoras de pesquisa envolvendo seres humanos. Secretaria de Assistência à Saúde. Conselho Nacional de Saúde. Programa Nacional de Doenças Transmissíveis/AIDS, 1997.

27. Pereira MG, Andrade ALSS. Avaliação de testes diagnósticos. In: Andrade ALSS \& Zicker F. Métodos de Investigação Epidemiológica em Doenças Transmissiveis. Organização Pan-Americana de Saúde: Fundação Nacional de Saúde, Brasilia 1: 9-29, 1997.

28. Pocock SJ. Clinical Trials - A practical Approach. A Wiley Medical Publication, 1983.

29. Rassi A, Luquetti AO. Therapy of Chagas disease. In Wendel S, Brener Z, Camargo ME, Rassi A, eds. Chagas disease (American trypanosomiasis): its impact on transfusion and clinical medicine. International Society of Blood Transfusion, Brazil' 92, Såo Paulo, Brazil 237-247. 1992

30. Rosser WW. Application of evidence from randomised controlled trials to general practice. Lancet 353:661-664, 1999.

31. Sackett DL. Bias in analytic research. J Chron Dis 32:51-63, 1979.

32. Silveira AC, Feitosa VR, Borges R. Distribuição de triatomíneos capturados no ambiente domiciliar, no período de 1975/83, Brasil. Rev Bras Malariol Doenças Trop 36:15-19, 1984.

33. Smith PG, Morrow RH. Ensayos de Campo de Intervenciones en Salud en Paises en Desarrollo: Una Caja de Herramientas. OPAS. Washington, 1998.

34. Sniderman Ad. Clinical trials, consensus conferences, and clinical practice. Lancet 354:327-330, 1999.

35. Souza AG, Valério-Waderley DM, Buralli GM, Andrade JCR. Consolidation of the control of Chagas'disease vectors in the state of São Paulo. Mem Inst Osvaldo Cruz 79(supl):125-131, 1984.

36. Spadeto AL. Eficácia do Benzonidazol no tratamento de crianças com infecção crônica recente pelo Trypanosoma cruzi após 6 anos de seguimento: Ensaio clinico aleatório, duplo-cego, placebo controlado. Goiânia [Tese de Mestrado em Medicina Tropical IPTSP/UFG], 1999

37. Viotti R, Vigliano C, Armenti H, Segura E. Treatment of chronic Chagas'disease with Benznidazole: Clinical and serologic evolution of patients with long-term follow-up. American Heart Journal 127:151-162, 1994.

38. Weel CV, Knottnerus JA. Evidence-based interventions and comprehensive treatment. Lancet 353:916-918, 1999.

39. World Health Organization - WHO. Technical Report Series. N. 850. Guidelines for good clinical practice (GCP) for trials on pharmaceutical products.Geneva, pgs 97-137, 1995. 